\title{
Molecular dynamics simulations on self-healing behavior of ionene polymer-based nanostructured hydrogels
}

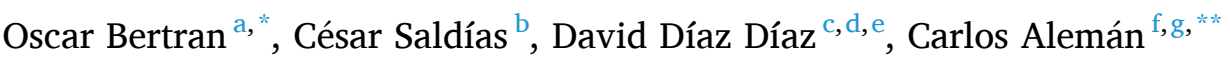 \\ ${ }^{a}$ Departament de Física EETAC, Universitat Politècnica de Catalunya, c/ Esteve Terrades, 7, 08860, Castelldefels, Spain \\ ${ }^{\mathrm{b}}$ Departamento de Química Física, Facultad de Química y Farmacia, Pontificia Universidad Católica de Chile, Macul, 7820436 Santiago, Chile

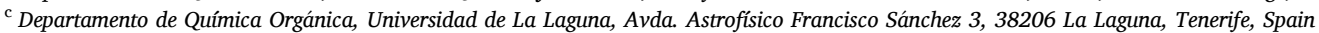 \\ ${ }^{\mathrm{d}}$ Instituto de Bio-Orgánica Antonio González, Universidad de La Laguna, Avda. Astrofísico Francisco Sánchez 2, 38206 La Laguna, Tenerife, Spain \\ ${ }^{\mathrm{e}}$ Institut für Organische Chemie, Universität Regensburg, Universitätsstr. 31, 93053 Regensburg, Germany \\ ${ }^{\mathrm{f}}$ Departament d'Enginyeria Química and Barcelona Research Center in Multiscale Science and Engineering, EEBE, Universitat Politècnica de Catalunya, Av Eduard \\ Maristany 10-14, 08019, Barcelona, Spain \\ ${ }^{\mathrm{g}}$ Institute for Bioengineering of Catalonia (IBEC), The Barcelona Institute of Science and Technology, Baldiri Reixac 10-12, 08028 Barcelona Spain
}

\section{A R T I C L E I N F O}

\section{Keywords:}

DABCO

Molecular dynamics

Polyelectrolyte hydrogels

Econstituted hydrogels

Self-healing mechanism

\begin{abstract}
A B S T R A C T
The microscopic mechanism accounting for the self-healing attribute of aromatic ionene-forming hydrogels derived from 1,4-diazabicyclo [2.2.2] octane (DABCO) and $N, N^{\prime}$-( $x$-phenylene)dibenzamide $(x=$ ortho-/meta-/ para-) is unknown. Interestingly, the self-healing property of such DABCO-containing hydrogels is largely dependent on the polymer topology, the ortho ionene being the only self-healable without adding oppositely charged species. In this work, Molecular Dynamics (MD) simulations have been conducted to evaluate the influence of the topology on ionene...ionene and ionene.*water interactions, as well as their effect on the selfhealing behavior. For this purpose, destabilized and structurally damaged models were produced for ionene hydrogels with ortho, meta and para topologies and used as starting geometries for simulations. These models were allowed to evolve without any restriction during MD production runs and, subsequently, the temporal evolution of ionene $\cdots$ ionene and water $\cdots$ ionene interactions was examined. Analysis of the results indicated that the ortho-isomer rapidly forms unique interactions that are not detected for other two isomers. Thus, in addition to the interactions also identified for the meta-and para-ionenes, the ortho-isomer exhibits the formation of strong intermolecular three-centered $(\mathrm{N}-) \mathrm{H} \cdots \mathrm{O}(=\mathrm{C}) \cdots \mathrm{H}(-\mathrm{N})$ hydrogen bonds, intramolecular planar sandwich $\pi-\pi$ stacking interactions and $\mathrm{Cl}^{-} \cdots \mathrm{N}^{+}$electrostatic interactions. Furthermore, the amount of intermolecular $\pi-\pi$ stacking interactions and the strength of water..polymer interaction are also influenced by the topology, favoring the stabilization of the ortho-ionene reconstituted hydrogels. Overall, the arrangement of the functional groups in the ortho topology favors the formation of more types of ionene...ionene interactions, as well as stronger interactions, than in the meta and para topologies.
\end{abstract}

\section{Introduction}

Polyelectrolytes in which the charged moiety (typically a quaternary ammonium cation) resides within the polymer backbone rather than on pendant group are called ionenes or ionene polymers [1]. In ionene polymers, the charge is high relative to other polycations and charge periodicity is easily controlled through the chemical structure. Ionenes are used in several important applications, as for example as gelators
[2-5], antimicrobials [6-9], components for organic electronics [10-12], and catalysts [13-15].

A particularly interesting family of ionene-forming hydrogels was derived from 1,4-diazabicyclo [2.2.2] octane (DABCO) and $N, N^{\prime}-(x$ phenylene)dibenzamide ( $x=$ ortho-/meta-/para-) [3]. As shown in Scheme 1, three different topologies, which correspond to the ortho-, meta- and para-substitution patterns, can be defined in these compounds. Previous experimental studies supported by computer

\footnotetext{
* Corresponding author.

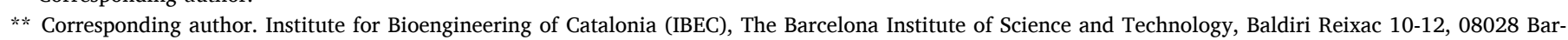
celona Spain.

E-mail addresses: oscar.bertran@upc.edu (O. Bertran), carlos.aleman@upc.edu (C. Alemán).
} 

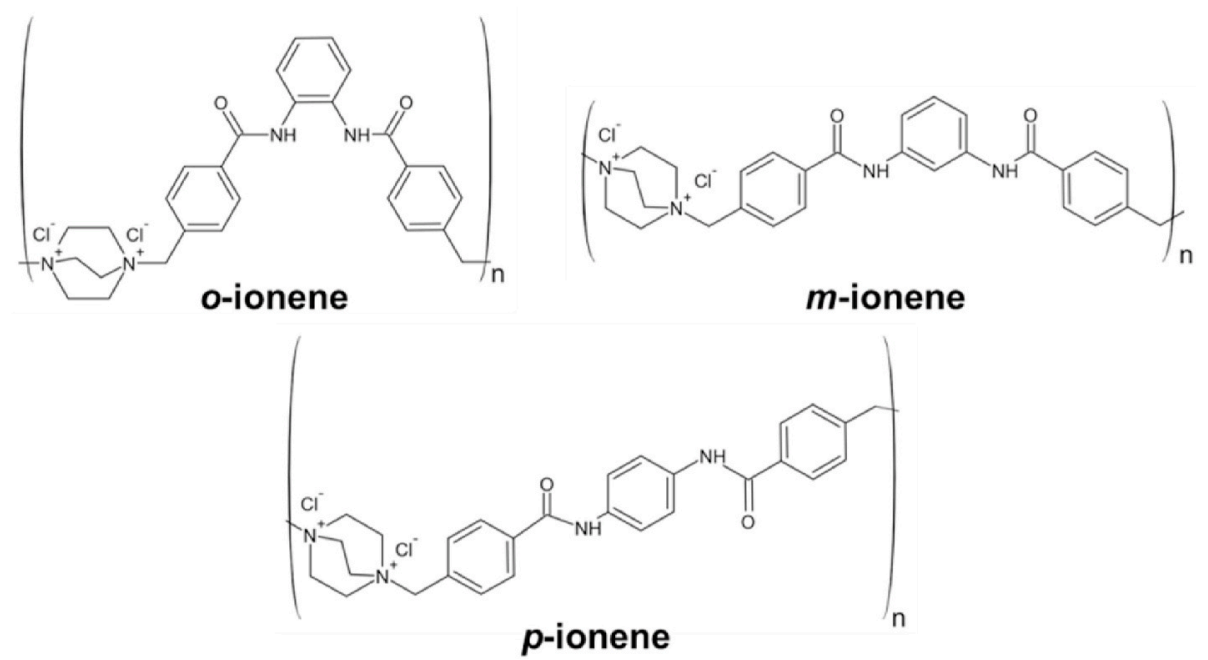

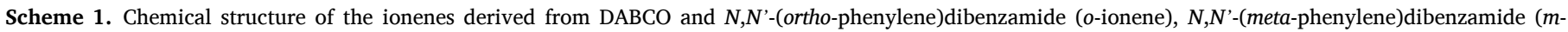
ionene) or $N, N^{\prime}$-(para-phenylene)dibenzamide ( $p$-ionene).

simulations demonstrated that the self-assembly and hydrogelation properties of such DABCO-containing ionenes are related with their topology $[3,16]$, which defines the separation among positive charges groups as well as of the position of the moieties able to participate in hydrogen bonding and $\pi-\pi$ stacking interactions.

The topology of these polymers defines their self-assembly properties in both pure water [2] and in aqueous solutions with different acidic $\mathrm{pH}$ and ionic strength [16]. In all cases the ortho-ionene (hereafter $o$-ionene) showed higher gelation ability (i.e. lower critical gelation concentration, higher gel-to-sol temperature and faster gelation) than the meta- and para-isomeric ionenes ( $m$-ionene and $p$-ionene, respectively) $[2,16]$. This was attributed to the capacity of the $o$-ionene in forming specific intermolecular interactions (i.e. hydrogen bonds and $\mathrm{N}-\mathrm{H} \cdots \pi$ interactions), distinctive $\pi-\pi$ intramolecular interactions, and to the binding action of $\mathrm{Cl}^{-}$counteranions stabilizing polymer $\cdots$ polymer interactions $[2,16]$. In the last years, several technological applications have proposed for these DABCO-containing ionenes. For example, they have been proposed as efficient systems for dye removal [17], as charge transfer catalysis [18], and as dopant agents for the electrodes of supercapacitors $[19,20]$. In addition such systems were found to exhibit a non-cytotoxic behavior [21].

In a very recent work, Díaz and coworkers [22] showed that the topology of the aromatic core determines the self-healing properties of $o-, m$ - and $p$-ionene. The $o$-ionene displayed greater shape-persistent, self-standing and self-healing properties than the $m$ - and $p$-ionene. Indeed, macroscopic self-healing capacity, which was observed immediately after cutting the hydrogel without the need of adding oppositely charged species, was only proved for the $o$-ionene. In this work we extend the rational of our previous studies, in which we established the relationships between the structure and properties of this family of hydrogels at the microscopy level $[2,16,19,20]$, to the self-healing capacity. For this purpose, the self-healing of $o$-, $m$ - and $p$-ionene has been studied using computer Molecular Dynamics (MD) simulations at molecular level using all-atom models. It should be remarked that, to the best of our knowledge, the self-healing mechanism of hydrogels was not studied before using atomistic computer simulations. Within this context, the utilization of modern computational approaches, like atomistic MD simulations, can be considered as important tools to investigate the molecular mechanism of self-healing process for small polymers. More specifically, the simulations reported in this work provide important information about the interactions involved in the self-healing of $o$-, $m$ - and $p$-ionene that, a priori, can hardly be obtained experimentally.

\section{Methods}

Molecular models used to simulate the reconstitution of $o-, m$ - and $p$ ionene hydrogels were prepared using a strategy that is described in the next section. MD simulations with such models, which contained more than a half million explicit atoms, were performed using NAMD 2.7 program [23]. MD is a computational tool that can capture time-dependent conformational changes at various conditions by calculating inter-atomic forces through solvent Newton's second law. More specifically, the atoms and molecules are allowed to interact for a fixed period of time, giving a view of the dynamic evolution of the system. Interactions are evaluated using a classical force-field with the corresponding force-field parameters.

All ionene parameters were extrapolated from AMBER03 libraries [24] with exception of the partial charges, which were taken from our previous work [3]. Water molecules were represented using the TIP3 model [25]. van der Waals interactions were computed using an atom pair cut-off distance of $14.0 \AA$. In order to avoid discontinuities in the potential energy function, non-bonding energy terms were forced to slowly converge to zero, by applying a smoothing factor from a distance of $12.0 \AA$ Al. Electrostatic interactions were extensively computed by means of Ewald summations. The real space term was defined by the van der Waals cutoff, while the reciprocal space was computed by interpolation into an infinite grid of points (particle mesh Ewald) with maximum space grid points being $1.0 \AA$ [26]. Bond lengths were constrained using the SHAKE algorithm [27] with a numerical integration step of $2 \mathrm{fs}$

After generation of starting geometries for the three ionenes using the strategy described in the next section, the whole systems were equilibrated and thermalized using the following four-step protocol: (1) 8000 steps of energy minimization using the Newton Raphson method but keeping fixed the polymer chains; (2) 2 ns NVT MD at $500 \mathrm{~K}$; (3) slow reduction of the temperature ( $10 \mathrm{ps} / \mathrm{K}$ ) from 500 to $298 \mathrm{~K}$ using NVT MD; and (4) 3 ns of NPT MD. The resulting structures were the starting point of $150 \mathrm{~ns}$ of NPT MD simulations, in which all atoms of the system were allowed to be mobile. For these productions runs, the pressure control was performed by means of the Nose-Hoover [28] piston combined with the piston fluctuation control implemented for Langevin Dynamics [29] for the temperature control. Pressure was kept at $1 \mathrm{~atm}$, the oscillation period was set at $0.2 \mathrm{ps}$ while the decay time was set at $0.06 \mathrm{ps}$ The piston temperature was set at the same value as the thermostat control, $298 \mathrm{~K}$, which used a damping coefficient of $1 \mathrm{ps}^{-1}$.

Optical microscopy images were taken from the hydrogel made of $o$ - 


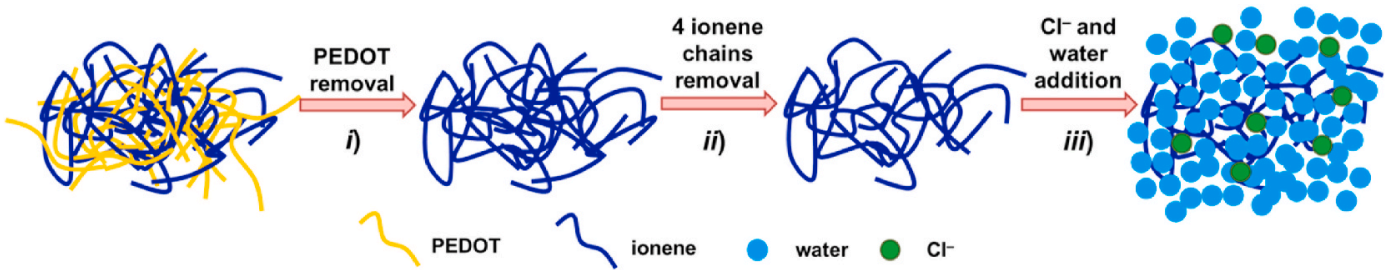

Scheme 2. Strategy used to construct the starting geometries for MD simulations.

ionene $(7.5 \% \mathrm{w} / \mathrm{v})$. A thin hydrogel film was formed on a glass microscope slide and the surface was damaged with a cutter. The self-healing process was monitored over $24 \mathrm{~h}$.

\section{Results and discussion}

Atomistic MD simulations mimicking the experimental conditions typically used for studying the self-healing capacity of hydrogels are limited by the length-and time-scales of such macroscopic assays. These frequently consist on the following steps [22,30]: 1) a pristine hydrogel sample (i.e. a cylinder or a strip) is cut in half; and 2) the two-halves are simply brought into contact and, if required, restorative liquid (e.g. pure water or an electrolytic aqueous solution) is contacted on the cut surface (e.g. by dropping or by immersion). After standing for a period of time, which can extend from a few minutes to some hours, the healed sample can be stretched without damage. Obviously, processes associated to

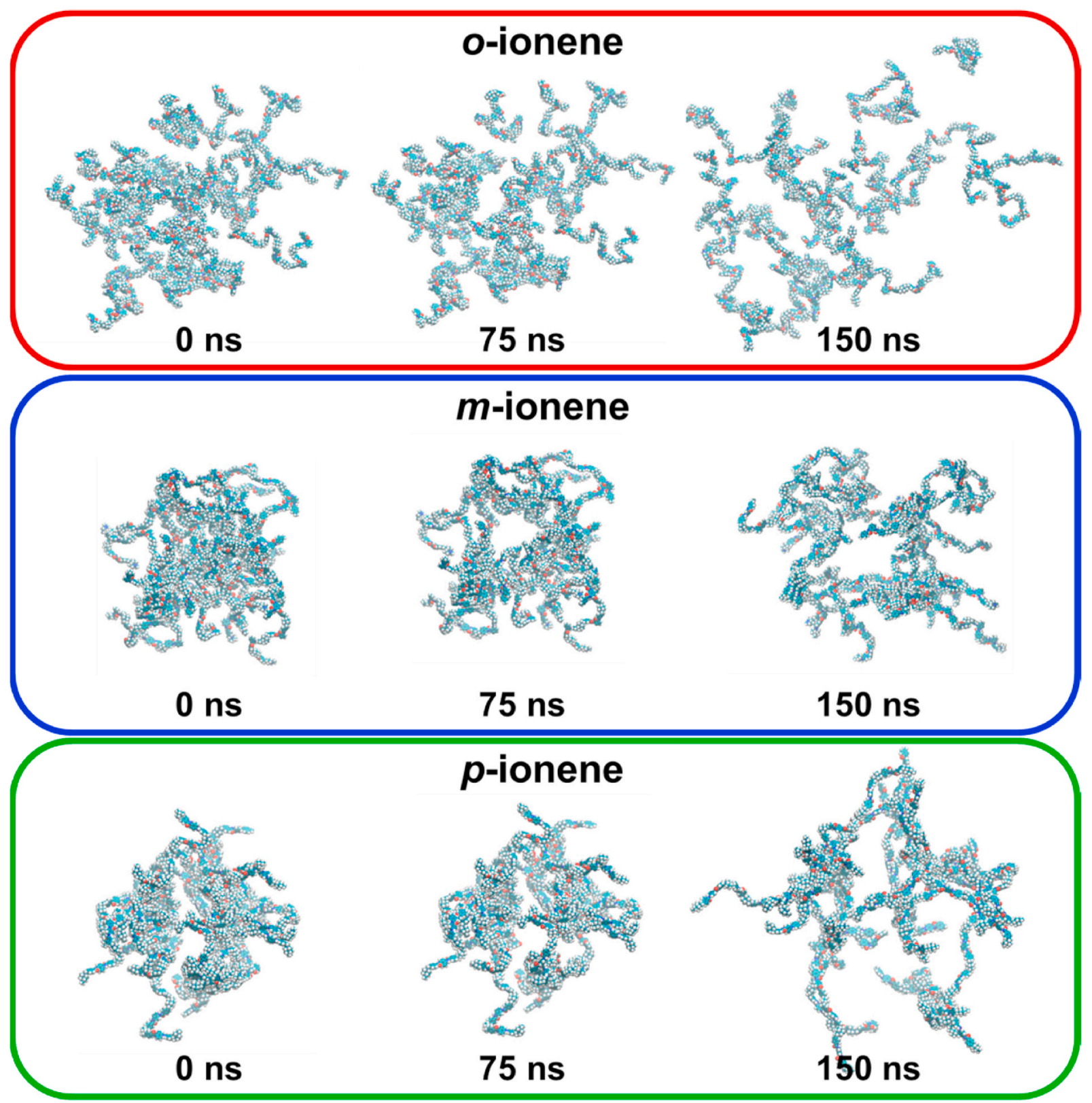

Fig. 1. Snapshots recorded at the beginning $(t=0 \mathrm{~ns})$, in the middle $(t=75 \mathrm{~ns})$ and at the end $(t=150 \mathrm{~ns})$ of the production trajectories for $o$-, $m$ - and $p$-ionene. 
(a)

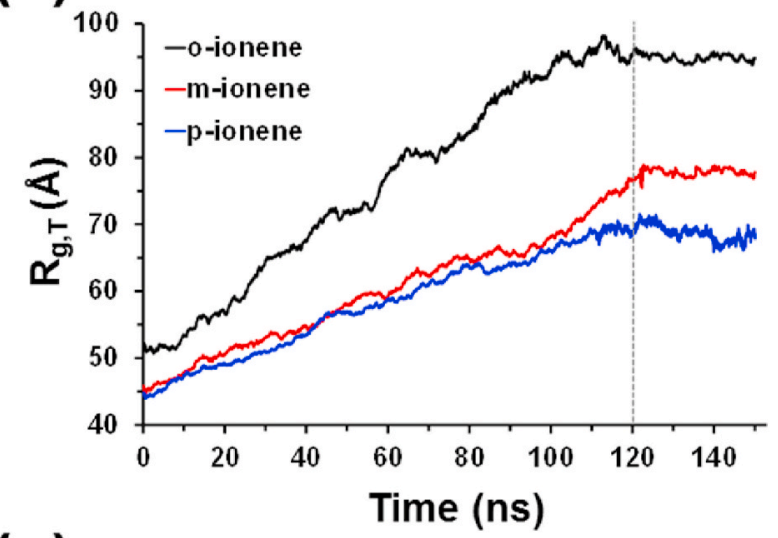

(c)

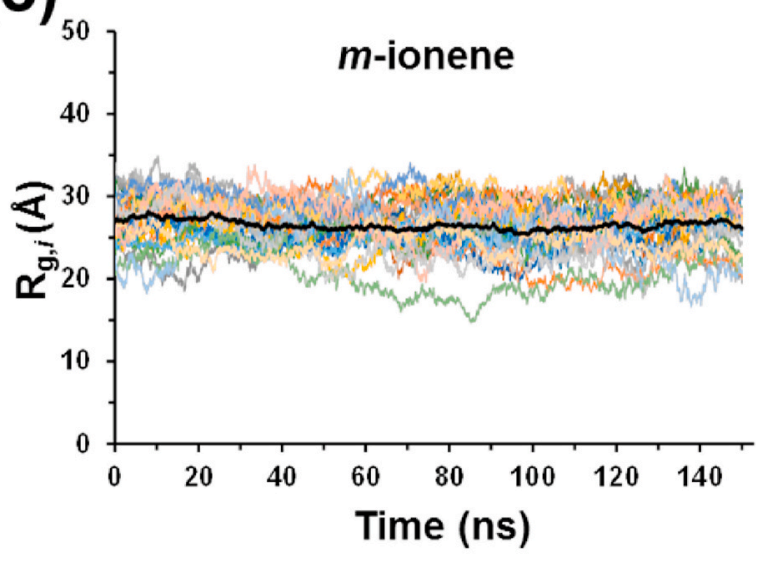

(b)

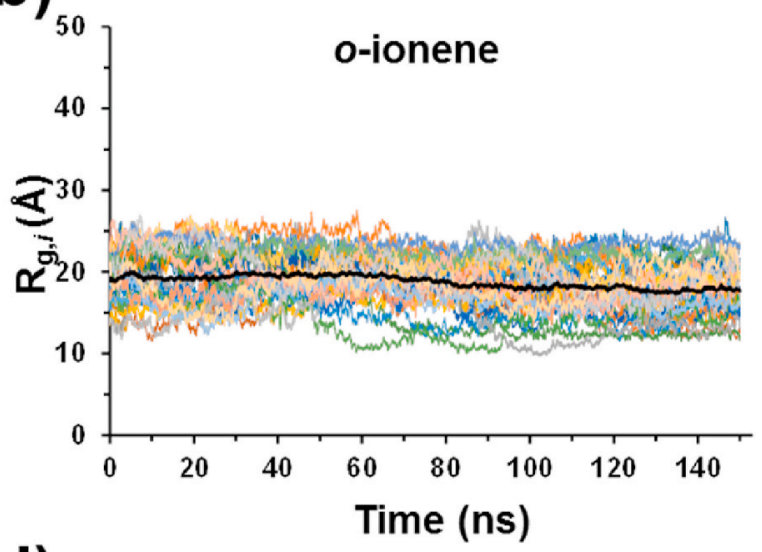

(d)

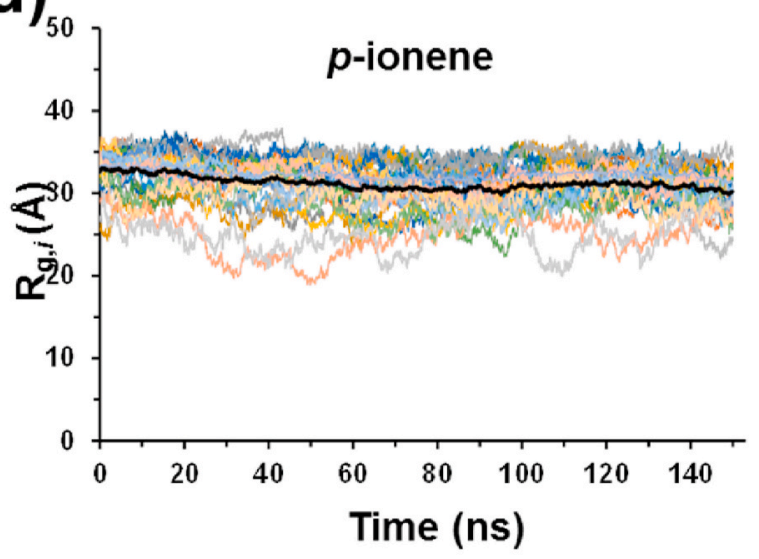

$(\mathbf{e})_{50}$

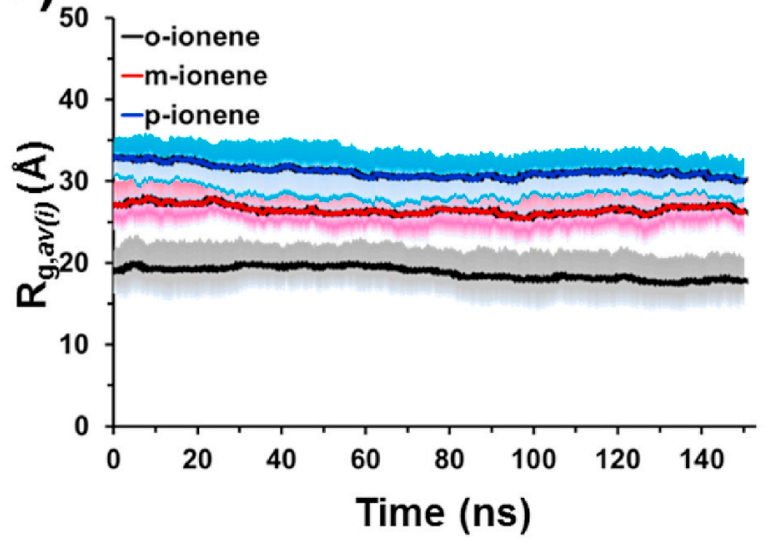

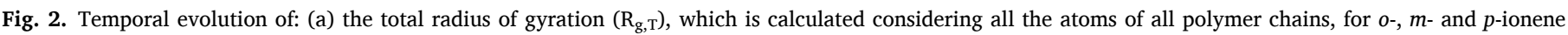

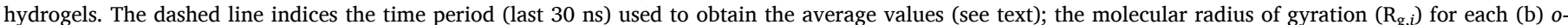

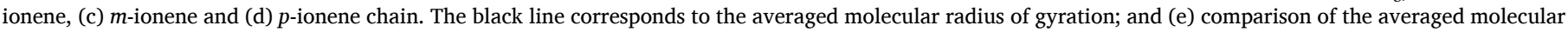
radius of gyration $\left(\mathrm{R}_{\mathrm{g}, a v(i)}\right)$ and the corresponding standard deviation (thin and thick lines, respectively) calculated for $o$-, $m$ - and $p$-ionene.

macroscopic cuts and healing processes that take at least several minutes are out both the length- and time-scales of molecular simulations.

In order to overcome the aforementioned limitations a strategy, which is sketched in Scheme 2, has been engineered to compare the behavior of the $o-, m$ - and $p$-ionene hydrogels when reconstituting. We started from the last snapshot recorded from production MD simulations reported on a recent study [19], which was devoted to study the influence of ionene topology on composites formed by ionenes and n-doped poly (3,4-ethyelenedioxythiophene) (PEDOT). Such trajectories were performed considering atomistic models formed by 48 PEDOT chains, each having 40 repeat units, and 32 ionene (o-, $m$ - or $p$-ionene) chains, each containing 6 repeat units. The size of the simulation box was $242 \times$ $161 \times 192,172 \times 214 \times 164$ and $182 \times 214 \times 168 \AA^{3}$ for the $o$-, $m$ - and $p$-ionene containing system, respectively [19]. Then, all PEDOT chains (step $i$ ) in Scheme 2) as well as the four ionene chains involving the greater number of intermolecular ionene ...ionene interactions (step ii) in Scheme 2) were removed from the simulation box. This caused not only a drastic destabilization of each system but also an important change in the structure and radial density profile of the 28 remaining ionene chains. Then, 336 chloride anions (i.e. 28 remaining ionene 
(a)

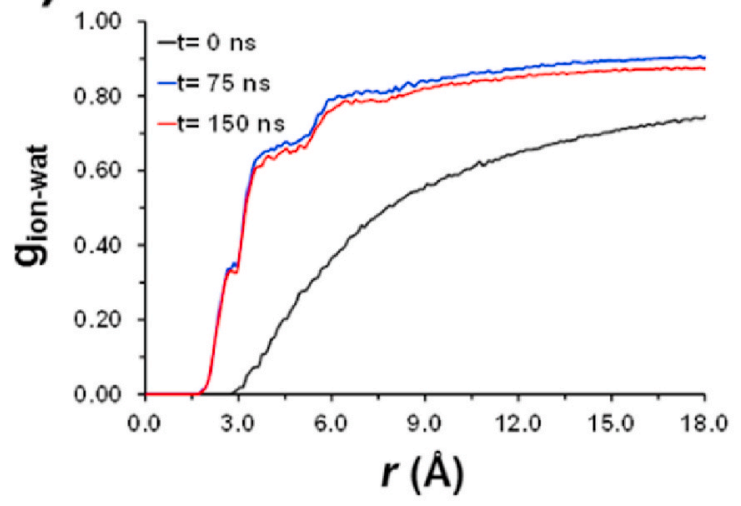

(b)

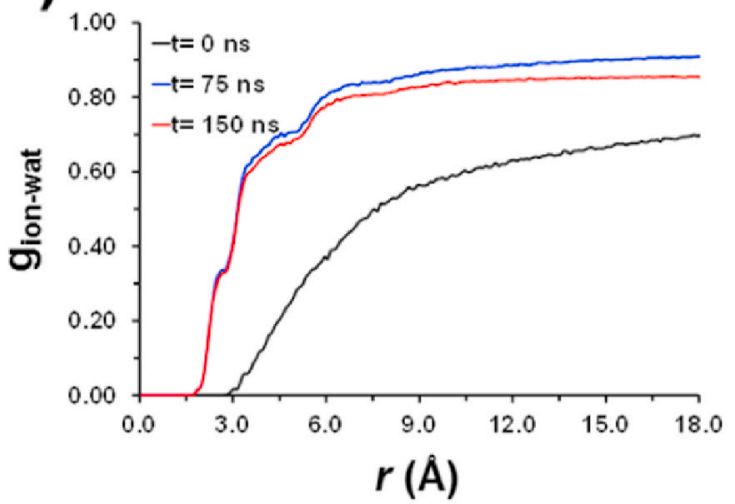

(c)

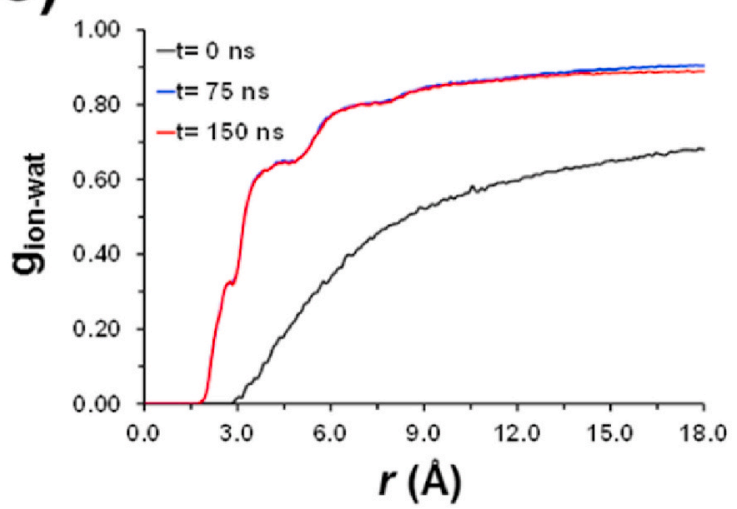

(d)
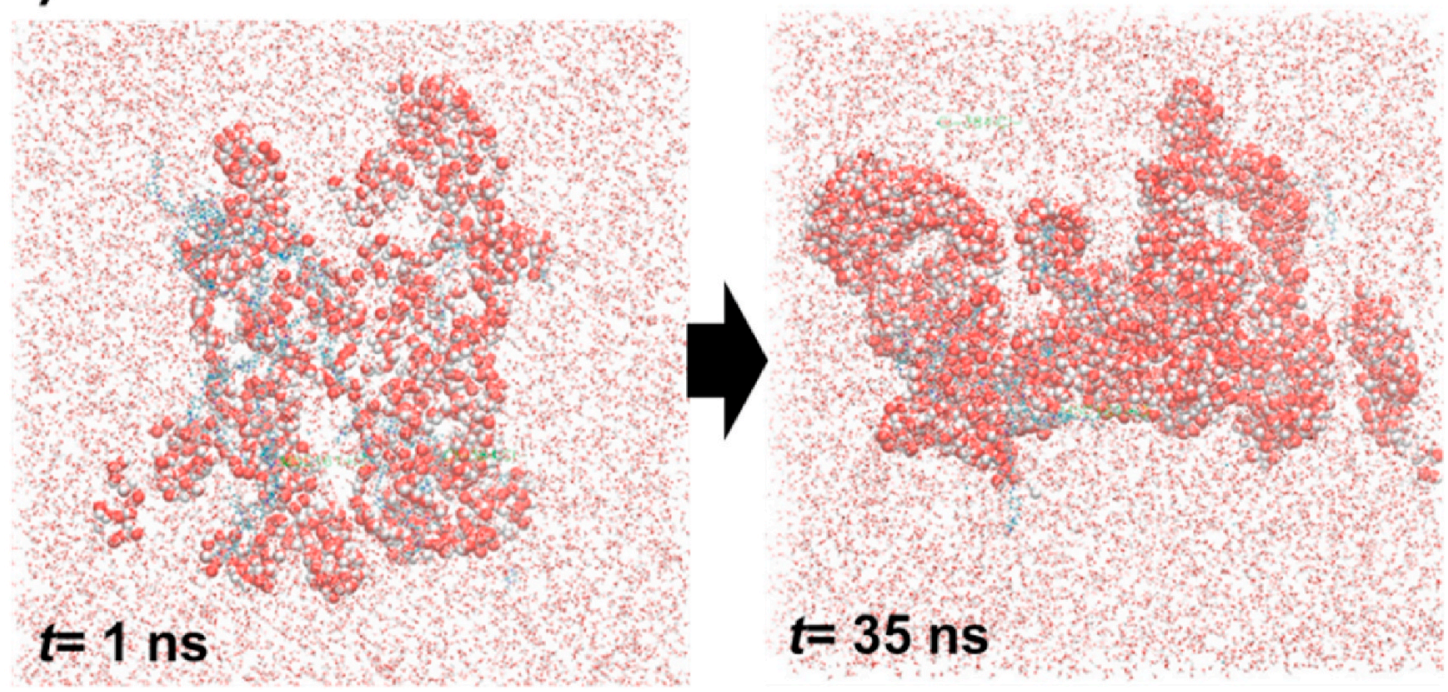

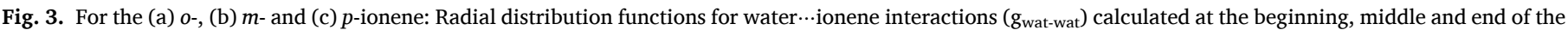

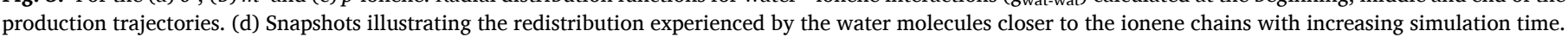

chains $\times 6$ repeat units per chain $\times 2$ positive charges per repeat unit) and water molecules (i.e. 215382, 180,034 and 186,563 water molecules for the $o$-, $m$ - and $p$-ionene, respectively) were added to the simulation box avoiding any overlap with the polymer atoms (step iii) in Scheme 2). It should be remarked that size of the simulation boxes was maintained at the aforementioned values along the whole process, the resulting systems involving a total of $675,374,551,330$ and 550,977 explicit atoms for the $o$-, $m$ - and $p$-ionene, respectively.
NVT MD simulations using protocol described in the Methods section were used to bring the systems at $298 \mathrm{~K}$, which remained constant during the production runs due to the coupling to the heat bath. After thermalization and equilibration, production NPT MD trajectories were run up to reach $150 \mathrm{~ns}$ In order to ensure the reproducibility of the tendencies discussed in this work, all simulations were performed in duplicate varying the starting points by changing 2 of the removed ionene chains. Results were very similar for the two sets of simulation 


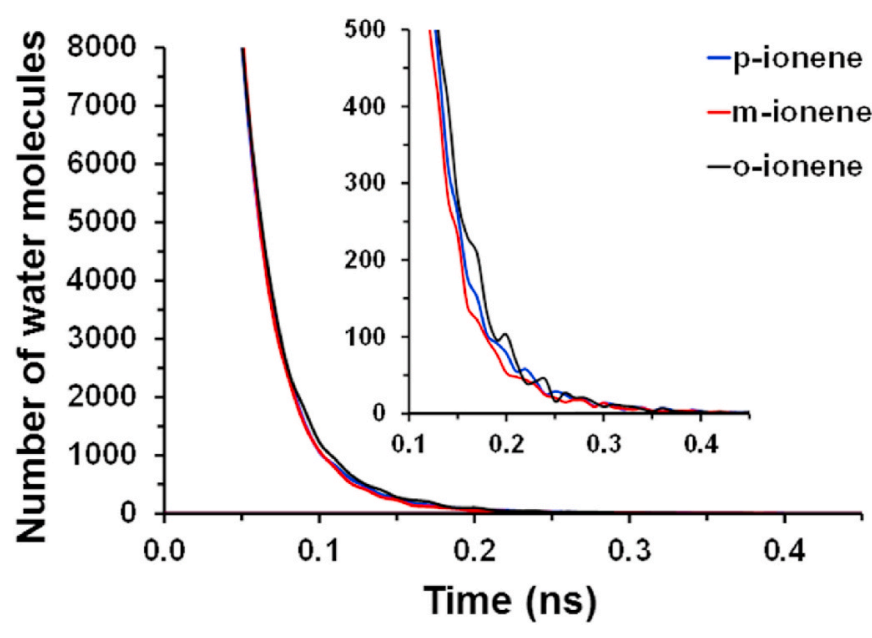

Fig. 4. Distribution of the residence times for water molecules located at the hydration shell of the studied ionenes. run for each studied ionene.

Fig. 1 compares the snapshots of the examined ionenes just after equilibration and thermalization (beginning of the production run) and after 75 and $150 \mathrm{~ns}$ In all cases, the structure evolves from a heterogeneous but relatively compact arrangement of ionene chains surrounded by water molecules to an open structure with water molecules occupying the regions between neighboring chains. The redistribution of ionene chains and the penetration of the water molecules is accompanied by a change in the size of the simulation box. At the end of the production run the size of the simulation box was $330 \times 118 \times 170,208$ $\times 124 \times 213$ and $203 \times 160 \times 177 \AA^{3}$ for the $o$-, $m$ - and $p$-ionene, respectively, which represent a volume contraction of $11.5 \%, 9.0 \%$ and $12.1 \%$ with respect to the initial value. Another important feature is the contact among neighboring ionene chains is apparently preserved in the open structures reached by the three systems, which has been attributed to the formation of specific interactions.

The structural transformation undergone by ionene aggregates is clearly evidenced when the temporal evolution of the total radius of gyration $\left(R_{g, T}\right)$, which is defined as the radius of gyration calculated considering the atoms of all polymer chains, and the radius of gyration of individual ionene chains is analyzed $\left(\mathrm{R}_{\mathrm{g}, i}\right)$. As shown in Fig. $2 \mathrm{a}$, the $\mathrm{R}_{\mathrm{g}}$, ${ }_{\mathrm{T}}$ of the three ionenes grows progressively until reach stable values at (a)

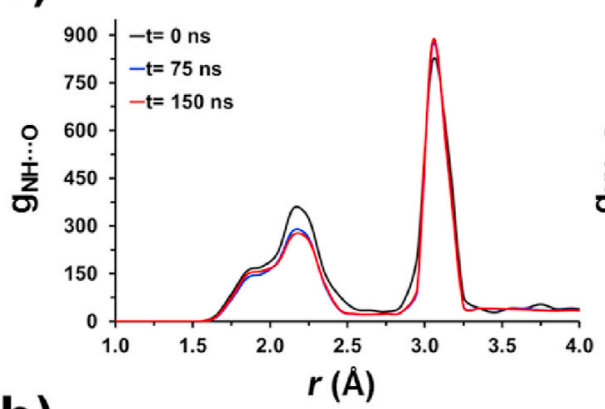

(b)

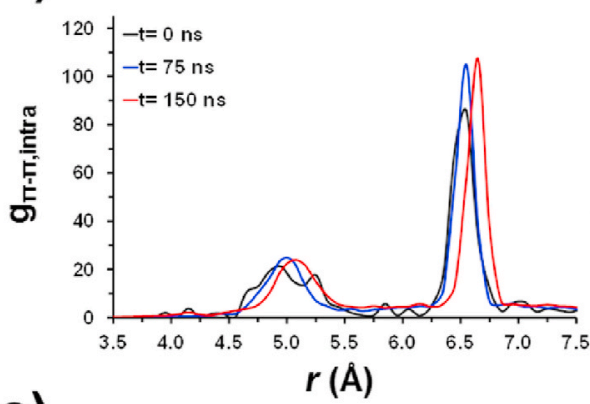

(c)

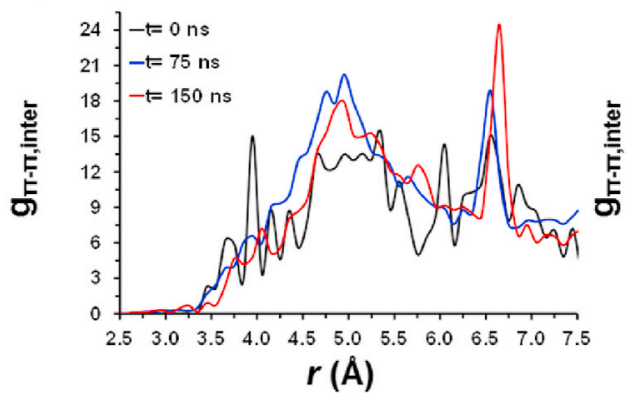

$m$-ionene
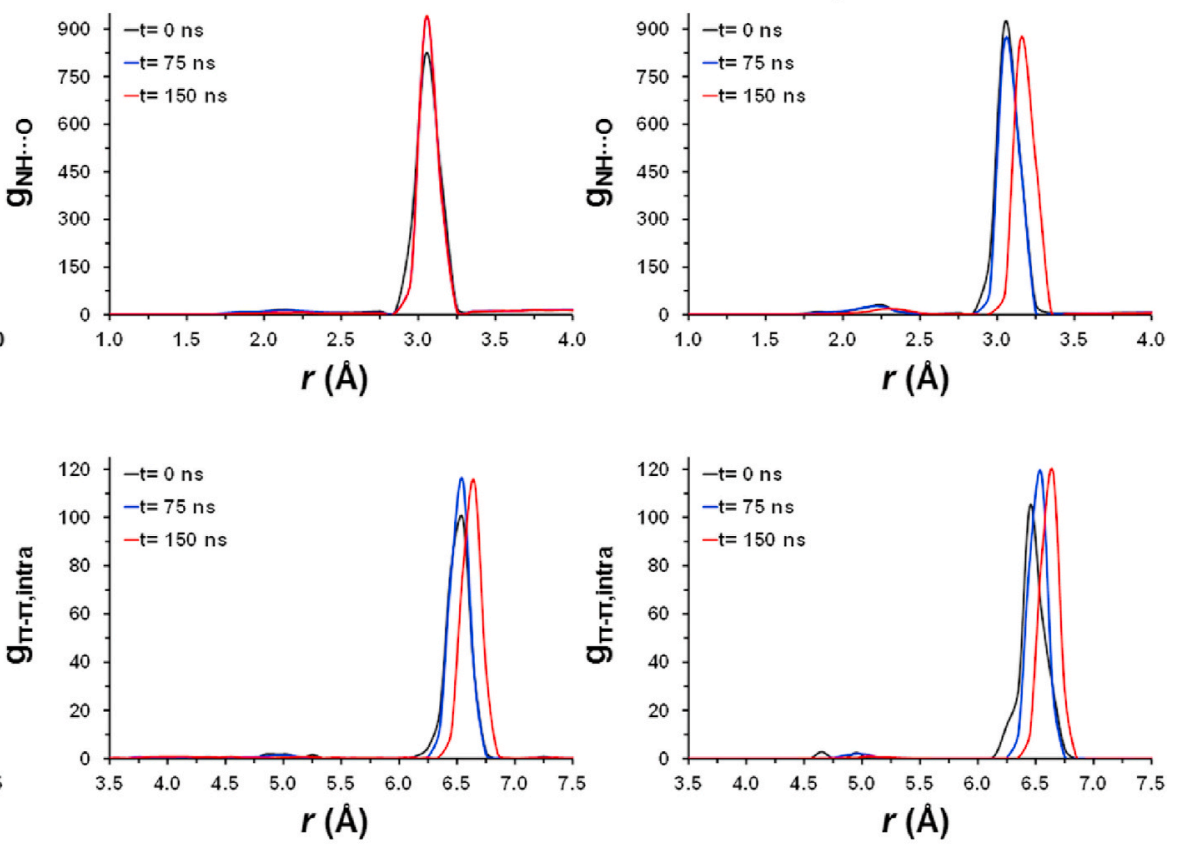

\section{p-ionene}

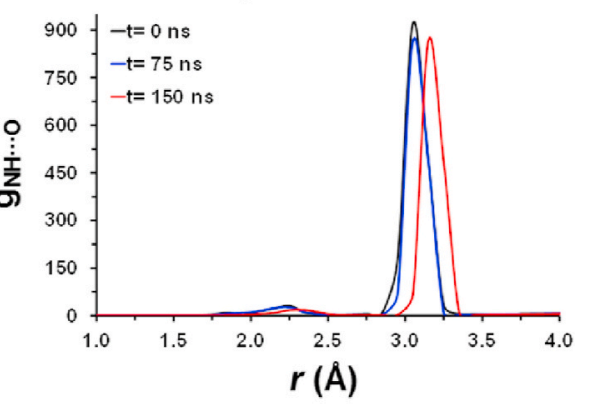

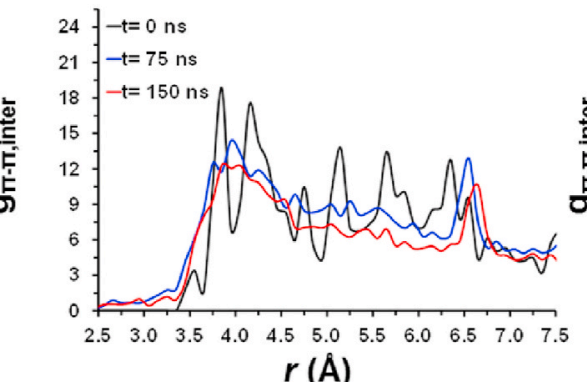

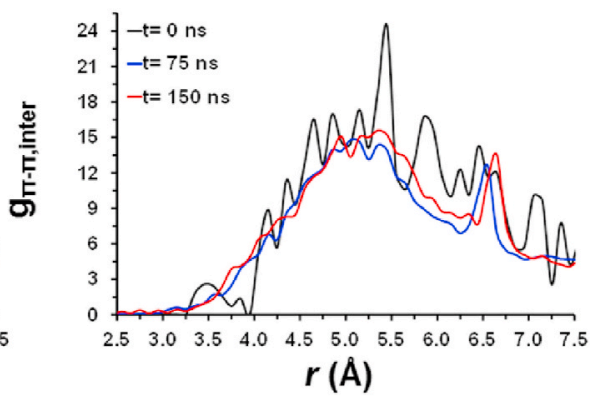

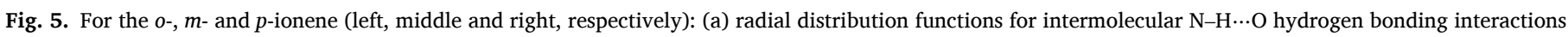

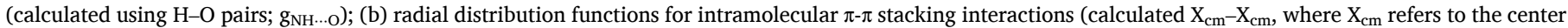

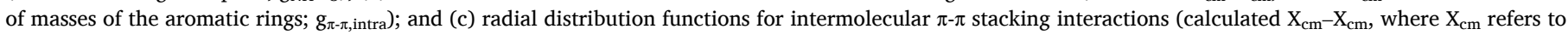

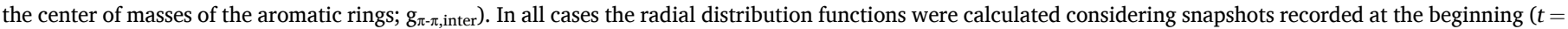
$0 \mathrm{~ns}$ ), in the middle ( $t=75 \mathrm{~ns}$ ) and at the end $(t=150 \mathrm{~ns})$ of the production trajectories. 


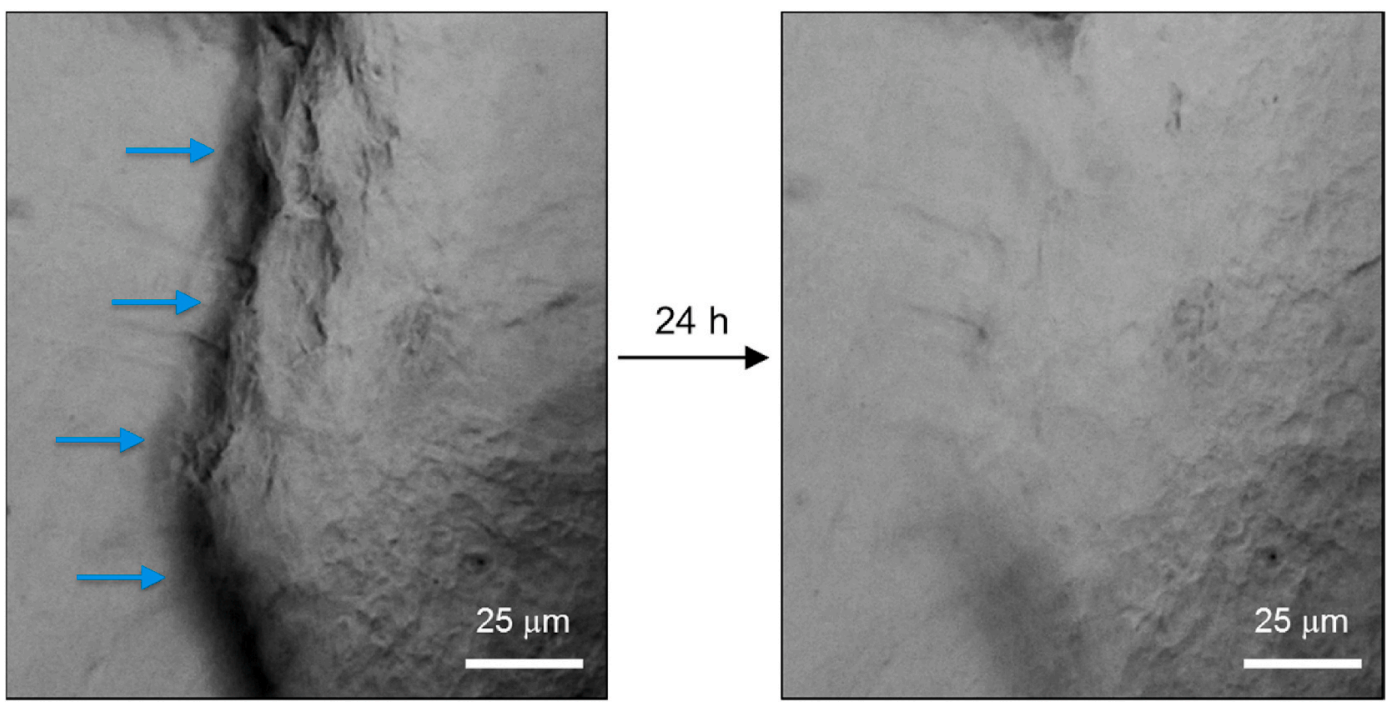

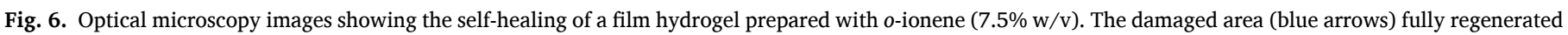
after $24 \mathrm{~h}$. (For interpretation of the references to colour in this figure legend, the reader is referred to the Web version of this article.)

around 100-120 ns, depending on the topology. The average value obtained considering the last $30 \mathrm{~ns}$ of the production run is $94.8 \pm 0.5$, $77.8 \pm 0.5$ and $68.2 \pm 0.9 \mathrm{~nm}$ for the $o$-, $m$ - and $p$-ionene, respectively. Compared with the values at the beginning of the production MD, such averages represent an increment of $82 \%, 59 \%$ and $53 \%$, respectively. Instead, inspection of the evolution $\mathrm{R}_{\mathrm{g}, i}$ for the 28 ionene chains $(i=1$ to 28 ) indicates that the individual polymer molecules do not undergo drastic changes in the shape, as is clearly evidenced in Fig. $2 \mathrm{~b}-\mathrm{d}$. This observation is independent of the ionene topology, indicating that all these systems are relatively rigid from a molecular point of view.

Fig. 2e compares the temporal evolution of average $\mathrm{R}_{\mathrm{g}, i}$ values and their corresponding standard deviations, which supports the previous statement. The standard deviations are similar for the three ionenes, indicating that the lack of molecular flexibility is not influenced by the topology. The molecular $\mathrm{R}_{\mathrm{g}}$ for the simulated polymer chains, which contain 6 repeat units, is $17.8 \pm 3.1,26.8 \pm 2.8$ and $30.7 \pm 2.5 \AA$ for the $o$-, $m$ - and $p$-ionene, respectively, that corresponds to $\sim 3.0,4.5$, and 5.1 $\AA$ 解eat unit.

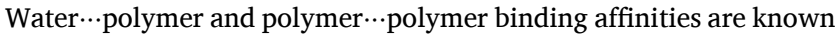
to play a decisive role not only in the formation of hydrogels but also in their structure and properties [31-33]. For physically crosslinked ionene hydrogels, we firstly hypothesized that the water $\cdots$ ionene interactions greatly affect the self-healing capacity. In order to examine such hypothesis the radial distribution functions for water $\cdots$ water ( $\left.g_{\text {wat-wat }}\right)$ and water $\cdots$ ionene pairs $\left(g_{\text {wat-Ion }}\right)$ of snapshots stored at the beginning $(t=0$ $\mathrm{ns})$, in the middle $(t=75 \mathrm{~ns})$ and at the end $(t=150 \mathrm{~ns})$ of the production trajectories are compared in Figures S1 and 3, respectively, for the three studied ionene topologies.

As expected, the $g_{\text {wat-wat }}$ profiles obtained for the $o-, m$ - and $p$-ionene are practically identical. In all cases a sharp and intense peak, which corresponds to the closest neighboring water molecules, is located $r=$ $2.75 \AA$ A . Besides, the profiles obtained for the initial snapshots become much smoother after some simulation, evidencing the relaxation and stabilization of the bulk water molecules in the reconstituted hydrogels. In any case, the $g_{\text {wat-wat }}$ curves obtained for the three ionene topologies are so similar that no information related with differences in the selfhealing mechanism can be extracted from them.

Although in all cases the shape of the $g_{\text {wat-Ion }}$ curve changes with the simulation time (Fig. 3), the three hydrogels exhibit a similar behavior in terms of water $\cdots$ ionene interactions. Initially, at $t=0 \mathrm{~ns}$, no peak is detected at $g_{\text {wat-Ion }}$, the shortest distance between water and the polymer chains occurring at $r \approx 3 \AA$. After this, the strength of water $\cdots$ ionene interactions increases and the shortest contact moves to $r \approx 1.8 \AA$ at $t=$
$75 \mathrm{~ns}$, reflecting the formation of a stable hydration shell around polymer chains that remains at $t=150 \mathrm{~ns}$ The formation of the stable hydration shell, which is illustrated in Fig. $3 \mathrm{~d}$ for the $o$-ionene, is completed after a few tenths of ns. However, this formation process as well as the characteristics of the hydration shell are independent of the ionene topology, as inferred from the similarity in the profiles at different simulation times (Fig. 3a-c).

Fig. 4 displays the residence time distribution of water molecules located in the ionenes hydration shell (i.e. considering water molecules at a distance lower of $4 \AA$ from any ionene atom). Although in general the water residence times are small in all cases (i.e. lower than $0.6 \mathrm{~ns}$ ), some differences are detected among the three ionenes, affecting the dynamics of the water molecules at the hydration shell. More specifically, the number of water molecules with residence times comprised between 0.15 and $0.60 \mathrm{~ns}$ is higher for the $o$-ionene than for the $m$ - and $p$ ionenes by $39 \%$ and $16 \%$, respectively. This feature suggests that the strength of water...polymer interaction is somehow influenced by the topology and decreases as follows: $o$-ionene $>p$-ionene $>m$-ionene. Despite of this, the diversity of behaviors found in the self-healing behavior of the three studied ionenes cannot be attributed to such small differences in water...polymer interactions.

To search for more distinctive features induced by the topology, capable of explaining the superior self-healing properties of the $o$-ionene compared to the $m$ - and $p$-analogues, ionene $\cdots$ ionene interactions were analyzed. For this purpose, the radial distribution functions for intermolecular $\mathrm{N}-\mathrm{H} \cdots \mathrm{O}$ hydrogen bonds $\left(\mathrm{g}_{\mathrm{NH} \ldots \mathrm{O}}\right)$, intramolecular $\pi-\pi$ stacking interactions $\left(g_{\pi-\pi, \text { intra }}\right)$ and intermolecular $\pi-\pi$ stacking interactions $\left(\mathrm{g}_{\pi-\pi, \text { inter }}\right)$ were calculated at the beginning, in middle and at the end of production trajectories ( $\mathrm{t}=0,75$ and $150 \mathrm{~ns})$.

The $g_{\mathrm{NH} \cdots \mathrm{O}}$ profiles for the $o$-, $m$ - and $p$-ionene are compared in Fig. 5a. Interestingly, the profiles obtained for the $o$-ionene (Fig. 5a, left) reveal a distinctive broad peak that extends from $r=1.8 \AA$ to $r=2.4 \AA$, indicating that the $o$-ionene is the only able to form strong intermolecular hydrogen bonds. Such interactions seem to be present during the whole trajectory, even though the intensity of the peak decreases slightly with the simulation time. In addition, a sharp peak that increases in the second half of the trajectory is observed at $r=3.0 \AA$. This weaker interaction is also observed for the $m$-ionene (Fig. 5a, middle). Regarding the $p$-ionene, it is worth noting that the strength of such interaction becomes weaker in the second half of the trajectory, as reflects the shift in the position of the peak from $r=3.0$ to $r=3.3 \AA$ (Fig. 5a, right). Overall, analyses of intermolecular hydrogen bonds reveal several distinctive features, which depend on the topology. 

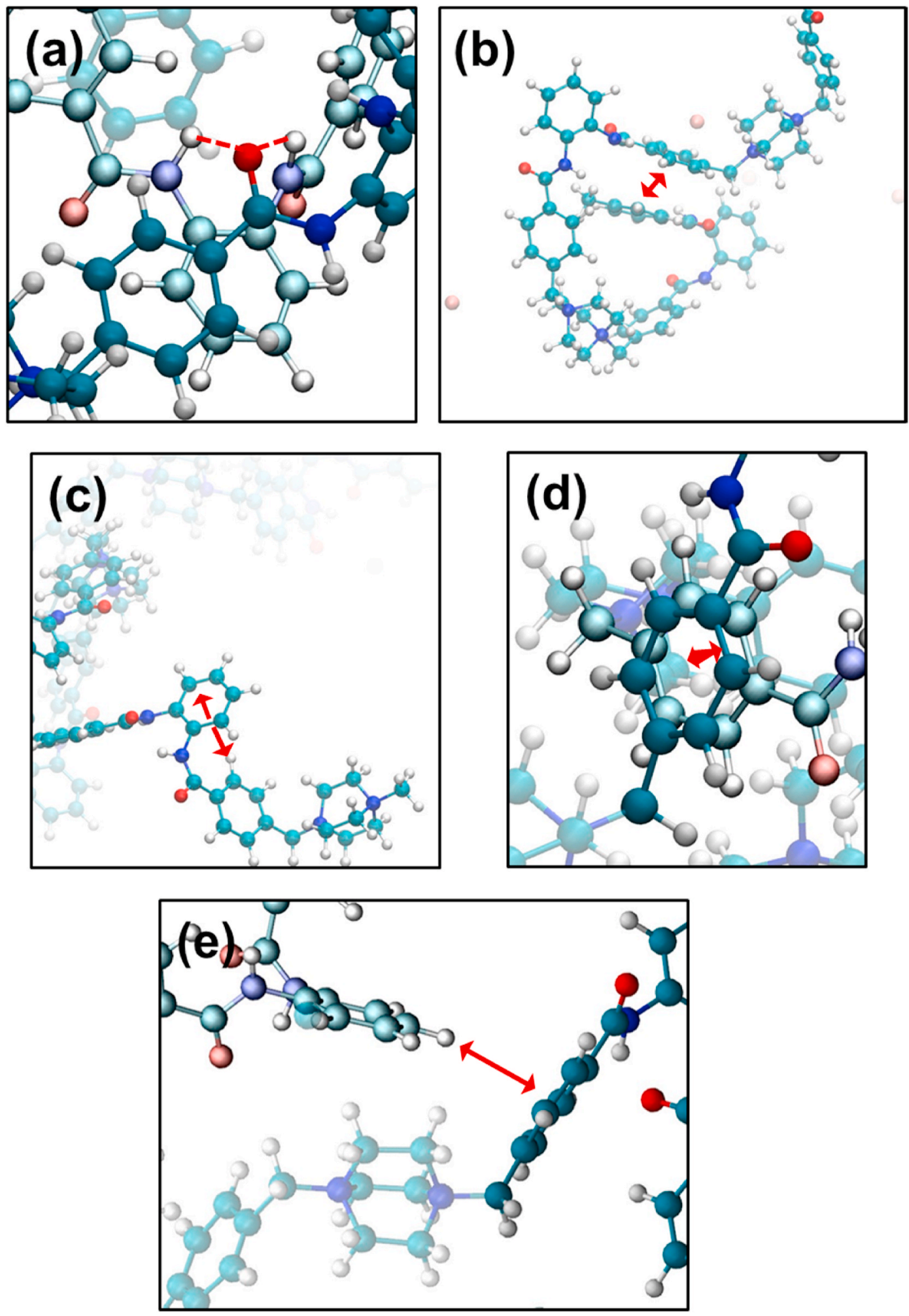

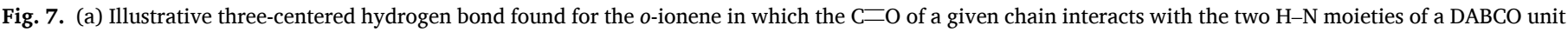

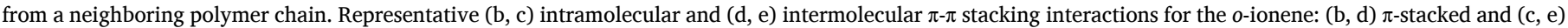
T-shaped configurations. The stacked interaction in (b) and (d) is formed between the two close aromatic rings arranged in parallel.

According to this kind of interactions, the reconstitution of hydrogels is favored following this order $o$-ionene $>m$-ionene $\gg p$-ionene, which is agreement with experiments [22]. Optical micrographs displayed in Fig. 6 illustrates the self-healing of the $o$-ionene hydrogel, which is completely reconstitution after $24 \mathrm{~h}$.

The two peaks identified for the $o$-ionene has been associated to the abundant three-centered or bifurcated $(\mathrm{N}-) \mathrm{H} \cdots \mathrm{O} \quad(=\mathrm{C}) \cdots \mathrm{H} \quad(-\mathrm{N})$ hydrogen bonds identified in the MD trajectory. This kind of interaction, which has been recently analyzed for proteins and polypeptides [34], is easily accommodated by the polymer bearing the ortho topology. Thus, the most usual geometry for three centered hydrogen bonds in the $o$-ionene involves a $\mathrm{C}=\mathrm{O}$ moiety from a polymer chain that is 
(a)

\section{o-ionene}

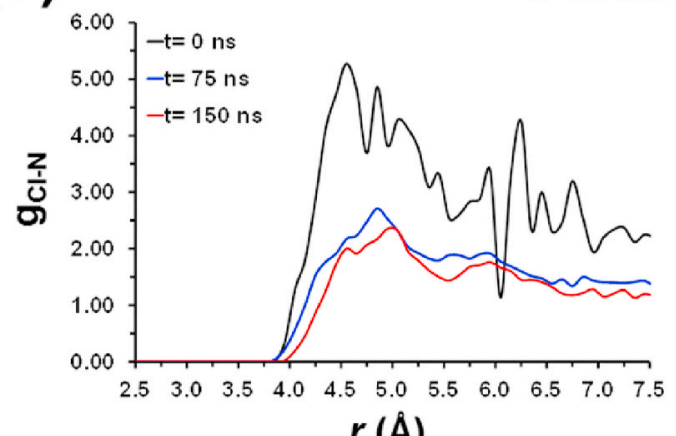

(b)
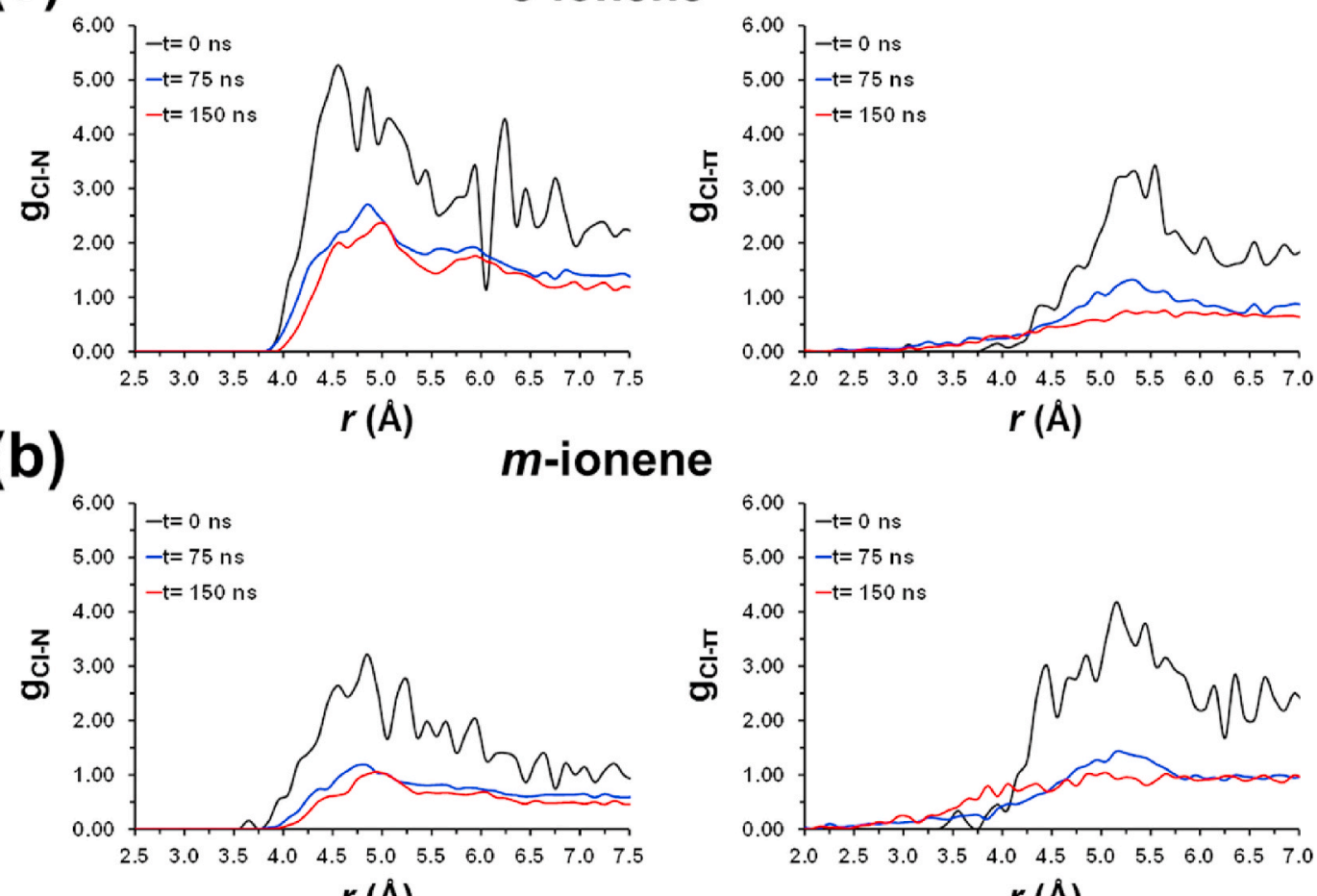

(c)

$r(\AA)$
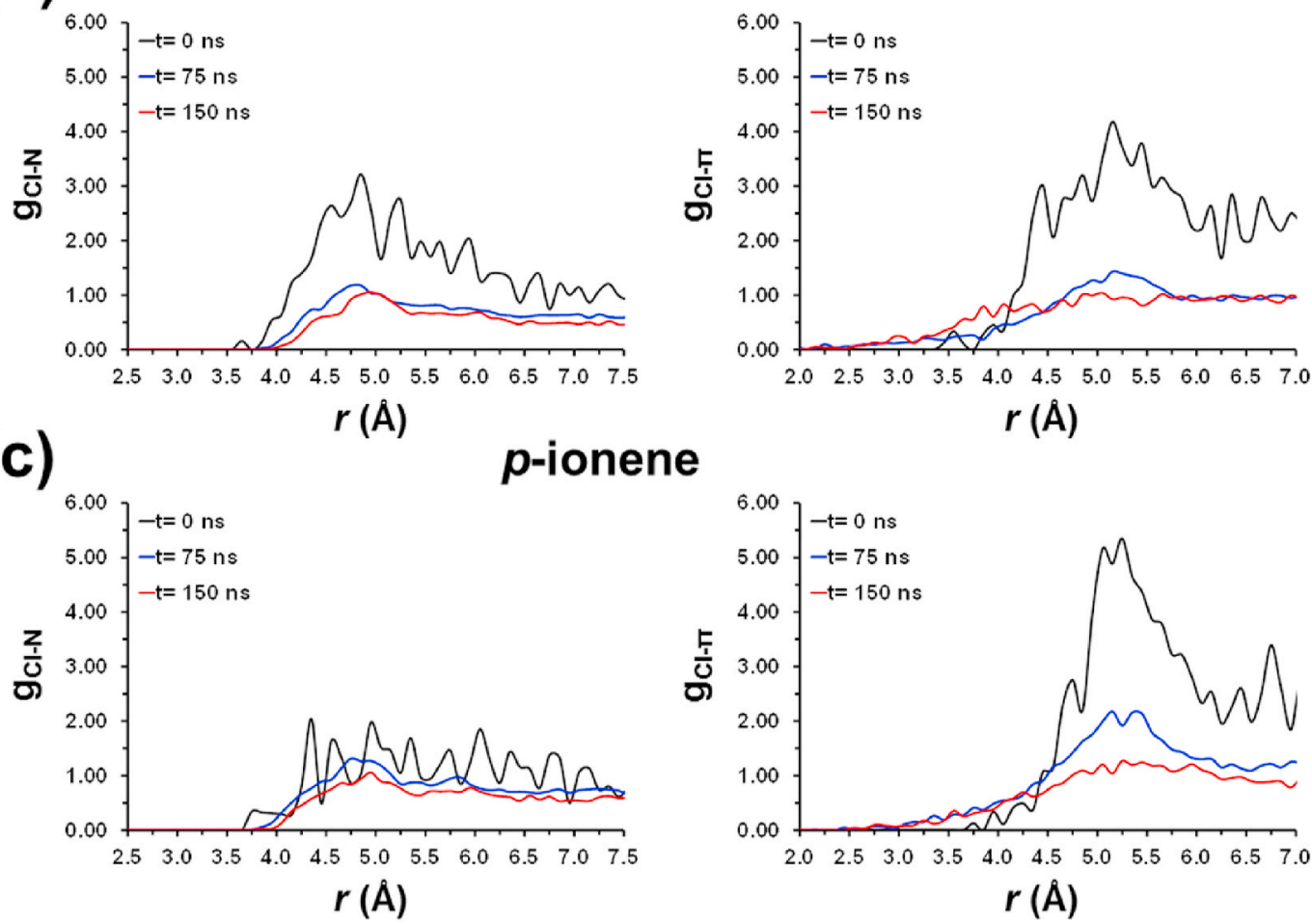

p-ionene

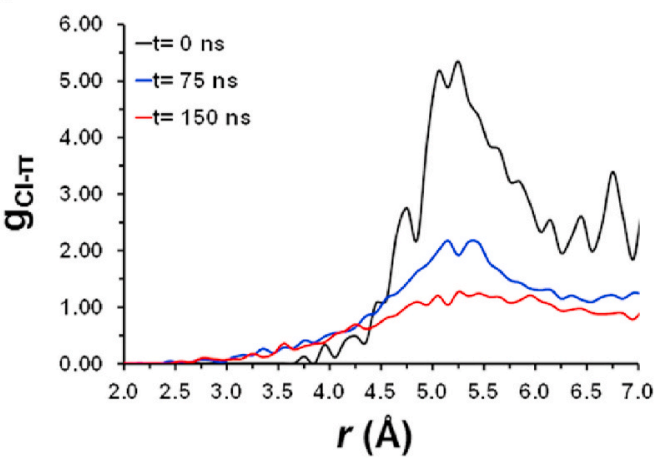

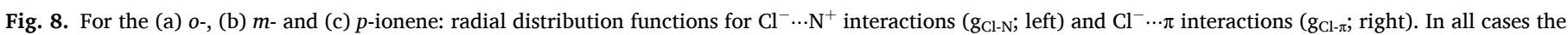

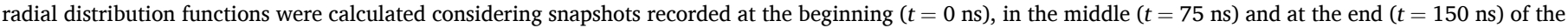
production trajectories. The $\mathrm{g}_{\mathrm{Cl}-\pi}$ profiles have been calculated using center of masses of the aromatic rings.

simultaneously interacting with the two $\mathrm{H}-\mathrm{N}$ groups of a given ortho-DABCO unit from a neighboring polymer chain, as is illustrated in Fig. 7a for a representative interaction. Instead, simple two-centered hydrogen bonds largely predominate for the $m$ - and $p$-ionenes. Indeed, the formation of bifurcated hydrogen bonds becomes much more difficult when the distance between the two $\mathrm{H}-\mathrm{N}$ moieties emerging from the DABCO unit increases, as occurs for the $m$ - and $p$-ionene. Figure S2 shows representative hydrogen bonds for the latter two polymers.

The $\mathrm{g}_{\pi-\pi \text {,intra }}$ profile calculated for the $o$-ionene at $t=0 \mathrm{~ns}$ (Fig. $5 \mathrm{~b}$, left) shows a broad peak centered at $r \approx 4.9 \AA$ and a sharp peak at $r=6.5$ $\AA$, which have been associated to $\pi-\pi$ stacking interactions involving planar sandwich ( $\pi$-stacked) and pseudo-perpendicular T-shaped configurations, respectively [35]. However, these interactions become slightly weaker with increasing simulation time, a $\Delta r \approx 0.2 \AA$ being observed at $t=150 \mathrm{~ns}$ The presence of intramolecular planar interactions is negligible for both the $m$ - and $p$-ionene (Fig. $5 \mathrm{~b}$, middle and right), all interactions showing a T-shaped configuration. However, as occurred for the $o$-ionene, the interactions become weaker with increasing simulation time, this effect being more pronounced for the $p$-ionene $(\Delta r \approx 0.4 \AA)$ than for the $m$-ionene $(\Delta r \approx 0.2 \AA)$. Fig. $7 \mathrm{~b}$ and c displays representative $\pi$-stacked and T-shaped interactions detected between aromatic rings of the same $o$-ionene chain at the end of the MD trajectory.

Inspection of the $g_{\pi-\pi, \text { inter }}$ profiles show a similar evolution for the three studied ionenes (Fig. $5 \mathrm{c}$ ). In all cases the profiles with multiple peaks obtained at $t=0$ ns convert into a broad peak centered at $r \approx 4-5$ $\AA$, which corresponds to the $\pi$-stacked configuration (Fig. 7d), and a sharp peak at $r=6.5 \AA(t=75 \mathrm{~ns}) / 6.7 \AA(t=150 \mathrm{~ns})$, which has been attributed to the T-shaped configuration (Fig. 7e) [35]. As expected, intermolecular $\pi-\pi$ stacking interactions become more defined with increasing simulation time. Moreover, the amount of interaction increases for the $o$-ionene (Fig. $5 \mathrm{c}$, left), whereas this effect is not detected for the $m$-and $p$-ionenes (Fig. $5 \mathrm{c}$, middle and right, respectively). Overall, these results indicate that intra- and intermolecular $\pi$ - $\pi$ stacking interactions play a crucial role in the reconstitution of the studied ionenes, providing explanation to the influence of the topology on the self-healing capacity.

The interactions between $\mathrm{Cl}^{-}$counterions and ionene chains may 
also play a crucial role in the reconstitution and self-healing processes. The moieties from ionene molecules able to interact with $\mathrm{Cl}^{-}$anions are the positively charged $\mathrm{N}$ atoms of DABCO units $\left(\mathrm{Cl}^{-} \ldots \mathrm{N}^{+}\right.$electrostatic interactions) and the three aromatic rings of each repeat unit $\left(\mathrm{Cl}^{-} \ldots \pi\right.$ interactions). Fig. 8 displays the radial distribution functions for $\mathrm{Cl}^{-} \cdots \mathrm{N}^{+}\left(\mathrm{g}_{\mathrm{Cl}-\mathrm{N}}\right)$ and interactions $\mathrm{Cl}^{-} \ldots \pi$ interactions $\left(\mathrm{g}_{\mathrm{Cl}-\pi}\right)$ for snapshots stored at the beginning, in middle and at the end of the production runs. Inspection of the $\mathrm{g}_{\mathrm{Cl}-\mathrm{N}}$ profiles indicates that the three studied ionenes experience an important reduction in the amount of $\mathrm{Cl}^{-} \ldots \mathrm{N}^{+}$interactions with increasing simulation time. This feature is very remarkable for the $m$ - and $p$-ionenes since the profile with multiple peaks obtained at $t=0 \mathrm{~ns}$ converts into almost flat profiles at $t=75$ and 150 ns This transformation indicates than the amount of $\mathrm{Cl}^{-} \cdots \mathrm{N}^{+}$interactions is residual for the ionenes with meta and para topologies.

For the $o$-ionene, the profile at $t=0 \mathrm{~ns}$ evolves towards profiles with a well-defined broad peak centered at $r=5.0$ and an ill-defined peak at $r$ $\approx 6.1 \AA$. The first distance, which is larger that sum of the van der Waals radii of the corresponding atom types $\left(R_{\mathrm{Cl}}=1.735 \AA\right.$ and $R_{\mathrm{N}+}=1.55 \AA$ ), has been attributed to the electrostatic interaction of each $\mathrm{Cl}^{-}$with a closest $\mathrm{N}^{+}$atom of a $o$-DABCO unit, whereas the second has been associated to the distance of the same $\mathrm{Cl}^{-}$atom to the other $\mathrm{N}^{+}$of the same $o$ DABCO unit. Thus, the $o$-ionene still shows an appreciable amount of $\mathrm{Cl}^{-} \cdots \mathrm{N}^{+}$interactions after $150 \mathrm{~ns}$ (Figure S3), suggesting that such electrostatic contacts have also some influence on its self-healing capacity.

The $\mathrm{g}_{\mathrm{Cl}-\pi}$ profiles show that, independently of the ionene topology, $\mathrm{Cl}^{-} \ldots \pi$ interactions are abundant at $t=0 \mathrm{~ns}$, exhibiting a broad and intense centered peak at $r=5.0-5.5 \AA$. However, these interactions become much less abundant at $t=75 \mathrm{~ns}$ and practically disappears at $t$ $=150 \mathrm{~ns}$, flat profiles being obtained at the end of the production trajectories for the three ionenes. This temporal evolution of the $\mathrm{g}_{\mathrm{Cl}-\pi}$ profiles indicates that the $\mathrm{Cl}^{-} \ldots \pi$ interactions do not affect the reconstitution process of the DABCO-containing ionenes. Overall, results displayed in Fig. 8 indicate that $\mathrm{Cl}^{-}$anions prefer to interact with the $\mathrm{N}^{+}$ atoms from non-aromatic rings than with the $\pi$-cloud of aromatic rings. However, quantitative assessment of $\mathrm{Cl}^{-} \ldots \mathrm{N}^{+}$electrostatic interactions reveals that these are only significant for the $o$-ionene, evidencing that the evolution of the $\mathrm{Cl}^{-} \ldots$ polymer interactions during the reconstitution of the hydrogels is influenced by the topology.

\section{Conclusions}

The interactions that establish the self-healing property of the $o$ ionene have been characterized by means of MD simulations. For this purpose, stressed and unstable models atomistic models were created for the ortho, meta and para isomers using the molecular geometries of previously studied dry composites involving such ionenes and PEDOT. After introducing water molecules to hydrate the systems, the models were relaxed and NPT trajectories were run to propitiate the formation of stable models for the $o$-, $m$ - and $p$-ionene hydrogels. It was found the gelation process mainly occurred through a re-distribution of the polymer chains but without altering their molecular shapes, giving place to open structures in which polymer chains are connected through specific intermolecular interactions.

The main difference between the reconstituted $o-, m$ - and $p$-ionene hydrogels is that the former is capable of forming bifurcated intermolecular hydrogen bonds, intramolecular planar sandwich $\pi-\pi$ stacking interactions and $\mathrm{Cl}^{-} \cdots \mathrm{N}^{+}$electrostatic interactions, while the other two hydrogels are not. According to these results, we conclude that electrostatic interactions play a crucial role in the self-healing process due to their strength. However, the role of hydrogen bonds and $\pi-\pi$ stacking interactions cannot be neglected, as they only appear in the $o$-ionene, even though they are significantly weaker than the electrostatic ones. Moreover, other small differences, that involve the strength and/or amount of interactions (e.g. polymer $\cdots$ water and intermolecular $\pi-\pi$ stacking interactions), also favor the reconstitution of the ortho hydrogel in comparison to the meta and para ones.

Overall, MD simulations have highlighted interactions that are only exhibited by the reconstituted $o$-ionene hydrogel, explaining its selfhealing property. Result demonstrate that the polymer topology, which defines ability to interact of the molecular chains, is a very important factor for the self-healing capacity. Although molecular-level computational and theoretical studies about the self-healing property of polymer hydrogels are still almost non-existent, this works demonstrates these molecular simulation methods can be used not only to understand the behavior of known healing systems but also to design new systems with optimized self-healing behavior.

\section{Declaration of competing interest}

The authors declare that they have no known competing financial interests or personal relationships that could have appeared to influence the work reported in this paper.

\section{Acknowledgements}

Authors acknowledge received funding from MINECO/FEDER (RTI2018-098951-B-I00), the Agència de Gestió d'Ajuts Universitaris i de Recerca (2017SGR359). Funding support for the research of C.A. was received through the prize "ICREA Academia" for excellence in research through the Generalitat de Catalunya. C. S. thanks Proyecto Inserción Académica (VRA UC). D. D. D. thanks the Spanish Ministry of Science, Innovation and Universities for the Senior Beatriz Galindo Award (Distinguished Researcher; BEAGAL18/00166) and NANOtec, INTech, Cabildo de Tenerife and ULL for laboratory facilities.

\section{Appendix A. Supplementary data}

Supplementary data to this article can be found online at https://doi. org/10.1016/j.polymer.2020.123072.

\section{References}

[1] M.D. Cole, M. Sheri, C. Bielicki, T. Emrick, Perylene diimide-based ionene and zwitterionic polymers: synthesis and solution photophysical properties, Macromolecules 50 (2017) 7535-7542.

[2] S.S. Babu, V.K. Praveen, A. Ajayaghosh, Functional $\pi$-gelators and their applications, Chem. Rev. 114 (2014) 1973-2129.

[3] J. Bachl, D. Zanuy, D.E. López-Pérez, G. Revilla-López, C. Cativiela, C. Alemán, D. D. Díaz, Synergistic computational-experimental approach to improve ionene polymer-based functional hydrogels, Adv. Funct. Mater. 24 (2014) 4893-4904.

[4] M. Yoshida, N. Koumura, Y. Misawa, N. Tamaoki, H. Matsumoto, H. Kawanami, S. Kazaouni, N. Minami, J. Am. Chem. Soc. 129 (2007) 11039-11041.

[5] N. Koumura, H. Matsumoto, H. Matsumoto, M. Yoshida, Hydrogels based on surfactant-free ionene polymers with $\mathrm{N}, \mathrm{N}^{\prime}$-(p-Phenylene) dibenzamide linkages, Macromolecules 41 (2008) 8841-8846.

[6] A. Strassburg, J. Petranowitsch, F. Paetzold, C. Krumm, E. Peter, M. Meuris, M. Köller, J.C. Tiller, Cross-linking of a hydrophilic, antimicrobial polycation toward a fast-swelling, antimicrobial superabsorber and interpenetrating hydrogel networks with long lasting antimicrobial properties, ACS Appl. Mater. Interfaces 9 (2017) 36573-36582.

[7] S.P. Rogalsky, O.V. Moshynets, L.G. Lyoshina, O.P. Tarasyuk, Antimicrobial polycarbonates for biomedical applications, EPMA J. 5 (2014) A133.

[8] M.S. Ganewatta, M.A. Rahman, L. Mercado, T. Shokfai, A.W. Decho, T.M. Reineke, C.B. Tang, Facially amphiphilic polyionene biocidal polymers derived from lithocholic acid, Bioact. Mater. 2 (2018) 186-193.

[9] M. Sánchez-Jiménez, F. Estrany, N. Borras, B. Maiti, D.D. Díaz, L.J. del Valle, C. Alemán, Antimicrobial activity of poly(3,4-ethylenedioxythiophene) n-doped with a pyridinium-containing polyelectrolyte, Soft Matter 15 (2019) 7695-7703.

[10] C. Huang, S. Barlow, S.R. Marder, Perylene-3,4,9,10-tetracarboxylic acid diimides: synthesis, physical properties, and use in organic electronics, J. Org. Chem. 76 (2011) 2386-2407.

[11] Z. Hu, R. Xu, S. Dong, K. Lin, J. Liu, F. Huang, Y. Cao, Quaternisation-polymerized N-type polyelectrolytes: synthesis, characterisation and application in highperformance polymer solar cells, Mater, Horizons 4 (2017) 88-97.

[12] Y. Liu, M.D. Cole, Y. Jiang, P.Y. Kim, D. Nordlund, T. Emrick, T.P. Russell, Chemical and morphological control of interfacial self-doping for efficient organic electronics, Adv. Mater. 30 (2018) 1705976.

[13] Y.M.A. Yamada, Y.A. Uozumi, A solid-phase self-organized catalyst of nanopalladium with main-chain viologen polymers: $\alpha$-alkylation of ketones with primary alcohols, Org. Lett. 8 (2006) 1375-1378. 
[14] P. Ahamed, Md A. Haque, M. Ishimoto, Md M. Parvez, N. Haraguchi, S. Itsuno, Synthesis of chiral quaternary ammonium polymers for asymmetric organocatalysis application, Tetrahedron 69 (2013) 3978-3983.

[15] A.T.N. Berlamino, E.S. Orth, R.S. Mello, M. Medeiros, F. Nome, Catalytic nanoreactors for ester hydrolysis, J. Mol. Catal. A 332 (2010) 7-12.

[16] J. Bach, O. Bertran, J. Mayr, C. Alemán, D.D. Díaz, Aromatic ionene topology and counterion-tuned gelation of acidic aqueous solutions, Soft Matter 13 (2017) 3031-3041.

[17] E.S. Dragan, J. Mayr, M. Häring, A.I. Cocarta, D.D. Díaz, Spectroscopic characterization of azo dyes aggregation induced by DABCO-based ionene polymers and dye removal efficiency as a function of ionene structure, ACS Appl. Mater. Interfaces 8 (2016) 30908-30919.

[18] M. Tiffner, K. Zielke, J. Mayr, M. Häring, D.D. Díaz, M. Waser, Phase-transfer catalysis with ionene polymers, Chem. Sel. 1 (2016) 4030-4033.

[19] M.C. Saborio, O. Bertran, S. Lanzalaco, M. Haring, L. Franco, J. Puiggalí, D.D. Díaz, F. Estrany, C. Alemán, Isomeric cationic ionenes as n-dopant agents of poly(3,4ethylenedioxythiophene) for in situ gelation, Soft Matter 14 (2018) 6374-6385.

[20] M.C. Saborio, O. Bertran, S. Lanzalaco, M. Haring, D.D. Díaz, F. Estrany, C. Alemán, Cationic ionene as an n-dopant agent of poly(3,4-ethylenedioxythiophene), Phys. Chem. Chem. Phys. 20 (2018) 9855-9864.

[21] J. Mayr, S. Grijalvo, J. Bachl, R. Pons, R. Eritja, D.D. Díaz, Transfection of antisense oligonucleotides mediated by cationic vesicles based on non-ionic surfactant and polycations bearing quaternary ammonium moieties, Int. J. Mol. Sci. 18 (2017) 1139.

[22] M. Häring, S. Grijalvo, D. Haldar, C. Saldías, D.D. Díaz, Polymer topologycontrolled self-healing properties of polyelectrolyte hydrogels based on DABCOcontaining aromatic ionenes, Eur. Polym. J. 115 (2019) 221-224.

[23] J.C. Phillips, R. Braun, W. Wang, J. Gumbart, E. Tajkhorshid, E. Villa, C. Chipot, R. D. Skeel, L. Kale, K. Schulten, Scalable molecular dynamics with NAMD, J. Comput. Chem. 26 (2005) 1781-1802.

[24] Y. Duan, C. Wu, S. Chowdhury, M.C. Lee, G. Xiong, W. Zhang, R. Yang, P. Cieplak, R. Luo, T. Lee, J. Caldwell, J. Wang, P. Kollman, A point-charge force field for molecular mechanics simulations of proteins based on condensed-phase quantum mechanical calculations, J. Comput. Chem. 24 (2003) 1999-2012.
[25] W.L. Jorgensen, J. Chandrasekhar, J.D. Madura, R.W. Impey, M.L. Klein, Comparison of simple potential functions for simulating liquid water, J. Chem. Phys. 79 (1983) 926-935.

[26] A. Toukmaji, C. Sagui, J. Board, T.J. Darden, Efficient particle-mesh Ewald based approach to fixed and induced dipolar interactions, J. Chem. Phys. 113 (2000) 10913-10927.

[27] J.P. Ryckaert, G. Ciccotti, H.J.C. Berendsen, Numerical integration of the cartesian equations of motion of a system with constraints: molecular dynamics of n-alkanes, J. Comput. Phys. 23 (1977) 327-341.

[28] G.J. Martyna, D.L. Tobia, M.L. Klein, Constant pressure molecular dynamics algorithms, J. Chem. Phys. 101 (1994) 4177-4189.

[29] S.E. Feller, Y. Zhang, R.W. Pastor, B.R. Brooks, Constant pressure molecular dynamics simulation: the Langevin piston method, J. Chem. Phys. 103 (1995) 4613-4622.

[30] I. Babeli, G. Ruano, J. Casanovas, M.P. Ginebra, J. García-Torres, C. Alemán, Conductive, self-healable and reusable poly(3,4-ethylenedioxythiophene)-based hydrogels for highly sensitive pressure arrays, J. Mater. Chem. C 8 (2020) 8654-8667.

[31] V. Gun'ko, I.N. Savina, S.V. Mikhalovsky, Properties of water bound in hydrogels, Gels 3 (2017) 37.

[32] E. Su, M. Yurtsever, O. Okay, A self-healing and highly stretchable polyelectrolyte hydrogel via cooperative hydrogen bonding as a superabsorbent polymer, Macromolecules 52 (2019) 3257-3267.

[33] Z.Y. Lei, Q.K. Wang, S.T. un, W.C. Zhu, P.Y. Wu, A bioinspired mineral hydrogel as a self-healable, mechanically adaptable ionic skin for highly sensitive pressure sensing, Adv. Mater. 29 (2017) 1700321.

[34] D.J. Kuster, C. Liu, Z. Fang, J.W. Ponder, G.R. Marshall, High-resolution crystal structures of protein helices reconciled with three-centered hydrogen bonds and multipole electrostatics, Plos One 10 (2015) e0123146.

[35] F. Rodríguez-Ropero, J. Casanovas, C. Alemán, Ab initio calculations on $\pi$-stacked thiophene dimer, trimer, and tetramer: structure, interaction energy, cooperative effects, and intermolecular electronic parameters, J. Comput. Chem. 29 (2008) 69-78. 